\title{
44522 - HOW OFTEN DO ANESTHESIOLOGISTS REALLY CHECK THEIR MONITORS?
}

\author{
Simon Ford, British Columbia Childrens' Hospital, Vancouver, BC, Canada; \\ Simon Ford, British Columbia Childrens' Hospital; \\ Elina Birmingham, Department of psychology, University of British Columbia, \\ British; \\ G Dumont, Department of Electrical and Computer Engineering; \\ J Lim, British Columbia Childrens' hospital, British Columbia; \\ J Daniels, British Columbia Children's Hospital, British Columbia; \\ JM Ansermino, British Columbia Children's Hospital, British Columbia;
}

INTRODUCTION:

Anesthsiologists rely on monitoring systems in the operating room (OR) to assist in measuring and maintaining a patient's clinical condition. These monitoring displays distance the clinician from direct contact with the patient. Therefore, an anesthesiologist's ability to ascertain information from a monitoring display is crucial to patient safety. We know very little about how anesthesiologists use monitors to gain information during surgery, and what other events in the OR affect information transfer. Previous studies used either a head mounted device (1) or an observer present in the OR who could interact with the clinician (2). These methods can affect the observed clinician's behavior and bias the observations. To accurately assess behavior, the anesthesiologist must be unaware of being observed. We report a novel, covert technique, to quantify frequency of monitor observation and concomitant organizational and behavioral actions. We also assess the clinicians behavior as the case progresses. METHODS:

Following IRB approval we obtained consent from 15 staff anesthesiologists allowing us to film them at any time during surgery without their knowledge. A remote controlled camera (1.3 megapixel webcam, Logitech Rightlight ${ }^{\mathrm{TM}}$ technology, Fremont, CA) was mounted on top of the monitor display panel (S/5 $5^{\mathrm{TM}}$, Datex-Ohmeda, Madison, WI, USA) in an OR prior to the start of the study, to allow clinician acclimatization. From each recorded case, 3 segments lasting 10 minutes were analyzed from the beginning (five minutes after induction), middle (mid-point of the case) and end of the case. The segments of video were analyzed for frequency, duration of monitor viewing by the anesthesiologist and concomitant activities.

RESULTS:

A total of 18 segments of video were analyzed from 6 cases. Monitor viewing behavior occurred in very short glances with a pattern that changed with case progression (Figure 1). Monitor viewing duration was significantly shorter at the end of the case compared to the beginning of the case (ANOVA with post hoc comparisons using Bonferroni correction, mean difference 1.0 seconds $95 \%$ CI $0.23-1.83$ ). On average, anesthesiologists 
looked at the monitor for only $4 \%$ of the total analyzed time. There was minimal overlap of task behaviors observed.

DISCUSSION:

We have developed a novel technique to assess the monitor display viewing behavior of anesthesiologists. Brief glances of the monitor display are performed. Only a few variables from the multiple variables displayed can be checked during a glance. Current monitor display design does not consider this method of use. Knowledge of this behavior can guide display design to maximize information transfer without altering anesthesiologist behavior. Adapting technology to current practice can enhance acceptance by clinicians, optimize utility and ultimately improve patient safety. REFERENCE LIST:

1)Anaesthesist 2002Mar;51(3):180-6.

2)Anesthesiology 1994Jan;80(1):77-92.

\begin{tabular}{|c|c|c|c|}
\hline & Beguraflice & Kate the & Eddfore \\
\hline 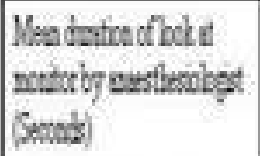 & 2330100 & 233304 & 130080 \\
\hline $\begin{array}{l}\text { Tazbobagtadss } \\
\text { (i) }\end{array}$ & 519 & 48 & 209 \\
\hline
\end{tabular}

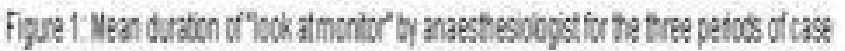

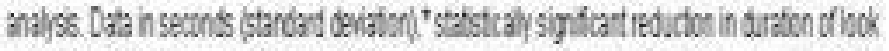
betwea to begring and end of cass, $P=0.012$ 